\title{
Investigation of the Efficiency of a Granular Filter Based on Cement Clinker
}

\author{
Rashid Sharapov ${ }^{1, *}$ \\ ${ }^{1}$ Moscow State University of Civil Engineering (National Research University), Moscow, 129337, \\ Russia
}

\begin{abstract}
The article describes the process of cleaning dusty gases. Attention is paid to the devices for fine cleaning of dusty gases. Attention is focused on the fact that the most simple and, at the same time, effective dust cleaning device is a granular filter. Its advantage is shown when cleaning dusty air from very aggressive devices, when working on hightemperature and aggressive gases. The prospects of granular filters, in which a cement clinker is used as a filter material, with a granule size of up to $10 \mathrm{~mm}$, are shown. The main causes of dust deposition on grains, filaments or fibers are presented. Various approaches to determining the empirical dependences of the dust span on the design parameters of the filter apparatus are presented. The criterion dependence of the cement dust span is presented, which approximates the results of the experiment. It allows you to determine the efficiency of the granular filter from its design and technological parameters.
\end{abstract}

\section{Introduction}

An increase in the production of various goods leads to the accumulation of various wastes, which pollutes our planet [1,2]. At the same time, a significant amount of energy is spent to reduce pollution and dispose of production waste. At the same time, the Russian Federation does not always meet the requirements of high environmental safety of production and lags behind the industrialized countries in addressing the provisions of the Kyoto Protocol [3].

Filters of various designs are used for fine cleaning of dusty gases [4]. However, these filters, while having a number of advantages, have negative sides. In the article, we will pay attention to granular filters, which, due to their simplicity of design and operation, are widely used in various industries [5-8].

Granular filters have a high efficiency, which in some cases exceeds $99 \%$ [9-12]. It is noted that granular filters are widely used in enterprises for the production of cement, in technological installations where the gas temperature is $400 \ldots 700{ }^{\circ} \mathrm{C}[13,14]$. In this article, we will consider the efficiency of granular filters, which use cement clinker particles as a filter layer.

\footnotetext{
*Corresponding author: ptdm_zavkaf@mail.re
} 


\section{Materials and Methods}

One of the main mechanisms of dust capture in the granular filter is the deposition of particles when the dusty gas flows around the grains of the bulk layer.

There are three causes of dust deposition on grains, filaments, or fibers: inertia, touch, and particle diffusion. The contribution of each of these causes is different and depends on the obstacle flow mode, which is determined by the Reynolds number $\mathrm{Re}=u d \rho / \mu$. There are two main flow modes: viscous, creeping flow $(\operatorname{Re} \leq 1)$, for which the gas viscosity is of decisive importance, and ideal potential flow ( $\operatorname{Re} \geq 1000)$, in which the effect of viscosity is negligible $[15,16]$.

It is established that the efficiency of inertial particle deposition is determined by the value of a dimensionless criterion - the Stokes number Stk, which characterizes the ratio of the inertia force to the aerodynamic drag force of the gas:

$$
\mathrm{Stk}=\frac{\delta^{2} \rho_{\mathrm{p}} u}{18 \mu d_{\mathrm{g}}} \mathrm{K}_{\mathrm{c}},
$$

где $u$ - скорость набегания потока запыленного газа, м/с; $\mathrm{K}_{\mathrm{c}}$ - поправка Каннингема where $u$ is the velocity of the dusty gas flow, mps; Kc is the Cunningham correction::

$$
\mathrm{K}_{\mathrm{c}}=1+\frac{2 \lambda}{\delta}\left(1,25+0,4 \exp \left(-0,55 \frac{\delta}{\lambda}\right)\right) \text {. }
$$

Here $\lambda$ is the free path length of the molecules (at $\mathrm{t}=20{ }^{\circ} \mathrm{C}, P=1,01 \cdot 10^{5} \mathrm{~Pa}, \lambda=$ $\left.6,5 \cdot 10^{-8} \mathrm{~m}\right)$.

Inertial particle deposition begins to appears at $\mathrm{Stk}>\mathrm{Stk}_{\mathrm{cr}}$, where $\mathrm{Stk}_{\mathrm{cr}}$ is the critical value of the Stokes number. For the viscous flow, $\mathrm{Stk}_{\mathrm{cr}}=0.6 \ldots 4.3$, for the potential $\mathrm{Stk}_{\mathrm{cr}}=$ $0.04 \ldots 0.125$. It is found that the efficiency of inertial particle deposition increases with increasing Stk, which can be caused by an increase in the particle size, their density, filtration rate, as well as a decrease in the grain size of the bulk layer. Inertial deposition is typical for particles with a size of $\delta>1 \mathrm{mkm}$.

If a sufficiently small particle does not collide with an obstacle, but passes close enough to it, then its deposition is possible due to contact (engagement) (Fig. 1).

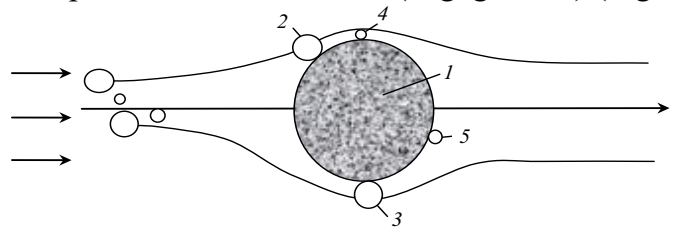

Fig.1. Scheme of dust particle deposition in a granular medium: 1-grain of the bulk layer; 2-particle deposited due to inertia; 3-particle deposited as a result of contact;4-particle deposited as a result of sedimentation; 5-particle deposited due to diffusion

The efficiency of particle deposition due to the tangency of nk depends on the ratio $R_{k}=$ $\delta / d$ and increases in the viscous flow regime. In this case,

$$
\eta_{\mathrm{K}}=R_{\mathrm{k}}^{2} \operatorname{Re}^{0.0625}
$$

The gravitational deposition (sedimentation) of dust particles in the granular layer is noticeable only at low filtration rates $(w \leq 0.02 \mathrm{mps})$. Its efficiency increases with increasing parameter $\mathrm{G}=\mathrm{Stk} / \mathrm{Fr}$, where $\mathrm{Fr}=u^{2} / 2 g d_{g}$ is the Froude number.

It is experimentally established that the efficiency of gravitational deposition in granular bulk filters increases significantly if the flow of dusty gas is directed vertically downwards, i.e. in the direction of gravity. 
Particles of submicron size $(\delta<<1 \mathrm{mkm})$ are involved in Brownian motion and migrate to the surface of the bulk layer grains due to molecular diffusion. The efficiency of the diffusion capture of particles increases with a decrease in the dimensionless Peclet criterion :

$$
\mathrm{Pe}=\frac{d u}{D}
$$

where $\mathrm{D}$ is the molecular diffusion coefficient of dust particles, which is determined by the Einstein formula:

$$
D=\frac{k \mathrm{TK}_{\mathrm{c}}}{3 \pi \mu \delta} .
$$

Here $k=1.38 \cdot 10^{-22} \mathrm{~J} / \mathrm{K}$ is the Boltzmann constant.

Electrical forces can have a noticeable effect on the process of capturing dust in granular layers. The grains of the filter layer can be electrified by friction, or polarized by an external electric field. Dust particles can be pre-charged using corona ionizers [17]. Thus, the deposition of particles in granular filters occurs as a result of the action of inertia, engagement, gravity, diffusion, and electric forces, so the relative passage of dust through the granular filter:

$$
K=Z_{\mathrm{f}} / Z_{\mathrm{b}},
$$

as well as the associated efficiency of cleaning dusty gases are functions of the criteria that determine the main mechanisms of dust capture discussed above, and some other dimensionless quantities $[18,19]$.

$$
\eta=1-K
$$

In particular, it is experimentally established that the degree of dust cleaning depends on the homochrony criterion $H \mathrm{o}=w \tau / d_{f}$, the ratio $Z_{i} / \rho$, as well as the geometric factors $\delta_{50} / \delta_{f}$ and $\delta_{f} / h$. Here $Z_{i}, Z_{f}-$ the initial and final dust concentration, $\mathrm{kg} / \mathrm{m}^{3} ; \mathrm{w}, \tau-$ the filtration rate and its duration, respectively; $h$ - the height (thickness) of the granular layer, $\mathrm{m}$.

Thus

$$
K=f\left(\operatorname{Re}, \mathrm{Stk}, H o, \delta_{50} / d_{\mathrm{i}}, \delta_{50} / h, \ldots\right) \text {. }
$$

When determining the type of dependence (8), it is necessary to take into account that the total efficiency of the granular filter should be more than the efficiency of any individual dust capture mechanism, but less than their sum. It should also be borne in mind that in real conditions, the action of all the mechanisms of dust capture is accompanied by a number of poorly studied side effects, such as adhesion, rebound of large particles from grains, secondary entrainment of deposited particles, etc.

In addition, the patterns of dust particle deposition discussed above relate only to the initial period of operation of the granular filter, i.e., to a clean granular layer. Over time, a layer of trapped particles - an autolayer-forms on the frontal surface of the granular layer, and the pore channels between the filter grains gradually become overgrown with dust deposits. This leads to a reduction in dust span and an increase in the hydraulic resistance of the layer.

These circumstances make it extremely difficult to theoretically deduce the dependence (8). Therefore, experimental methods for studying the processes of cleaning dusty gases with granular layers are brought to the fore.

Various approaches are used to deduce the empirical dependences of the dust slip on the properties of the filter nozzle, the properties of the dusty gas, and the filtration mode. 


\section{Results}

Thus, by processing the experimental data by the least squares method for a gravel bulk filter, the ratio is obtained [20]:

$$
K=\exp (-c(a+b \tau))
$$

where $a=0.877 \cdot 10^{-7}, b=2.57 \cdot 10^{-11}$-mpirical constants, $c$ - particle capture coefficient:

$$
c=\frac{h^{0,25} \delta_{50} \rho_{\mathrm{p}}}{w^{0,5} d_{\mathrm{g}}^{1,5} \mu} .
$$

Formula (10) is obtained for the following parameter range: $d_{\mathrm{g}}=1.5 \ldots 5 \mathrm{~mm}$; filtration rate $w=0.1 \ldots 0.3 \mathrm{mps} ; \delta_{50}=5 \ldots 30 \mathrm{mkm} ; \rho_{p}=2600 \ldots 3000 \mathrm{kgpm}^{3} ; \mu=(1.8 \ldots 2.5) \cdot 10^{-5} \mathrm{~Pa} \cdot \mathrm{s}$.

For the conditions of capturing cement dust $\left(\delta_{50}=30\right.$ microns ; $\rho_{p}=2600 \mathrm{kgpm}^{3} ; \mu=$ $\left.1.8 \cdot 10^{-5} \mathrm{~Pa} \cdot \mathrm{s}\right)$ with a bulk clinker layer $\left(h=0.15 \mathrm{~m}, d=5 \mathrm{~mm}, w=0.15 \mathrm{mps}, \mathrm{c} \cong 1.97 \cdot 10^{7}\right)$, the formula (9) takes the form:

$$
K=\exp \left(-1,97\left(0,877+2,57 \cdot 10^{-4} \tau\right)\right)
$$

According to the formula (11), the span of cement dust through the bulk clinker filter decreases from $17.8 \%$ at a clean layer $(\tau=0)$ to $2.9 \%$ after 1 hour $(3600 \mathrm{~s})$ after the start of filtration which is significantly higher than the observed values.

The formula included in the calculation method of bulk granular filters by N.M. Samokhvalov has a structure similar to (9) $[21,22]$ :

$$
K=\exp \left(-2,3 K \mathrm{e} \frac{h^{0,8}}{w}\left(1+K_{\mathrm{y}}\left(\frac{\tau}{\tau_{1}}\right)^{0,16}\right),\right.
$$

where $K \mathrm{e}=34500 \mu \delta_{50}^{0,25}(1-\varepsilon) /\left(\rho_{\mathrm{d}} d_{\mathrm{p}}^{2,05}\right)$ - dust particle capture efficiency coefficient, $K_{\mathrm{y}}=0,00132 P_{\mathrm{a}}^{n} /\left(\delta_{50} \rho_{\mathrm{d}} g \operatorname{tg} \alpha_{0}\right)-$ coefficient of dust removal from the granular layer, $\tau_{1} \geq 4,3 \cdot 10^{-6} \rho_{\mathrm{d}} / \delta_{50}$ - the time when the dusty stream is located in the granular layer. Here $\rho_{d}$ is the bulk density of dust, $\mathrm{kgpm}^{3}$ (for cement dust $\rho_{d}=1300 \mathrm{kgpm}^{3}$ ), $P_{\mathrm{a}}-$ autohesional strength of the dust layer, $\mathrm{Pa} ; n$ - the exponent depending on the value of $\mathrm{Pa}$ (for cement dust, $P_{a}=600 \mathrm{~Pa}, n=0.7 ; g-$ the acceleration of gravity; $\alpha_{0}$ - the angle of the natural slope of the dust, deg. (for cement dust, $\alpha_{0}=40^{\circ}$ ).

For the conditions of capturing cement dust with a bulk clinker layer, $\delta_{50}=30 \mathrm{mkm} ; h=$ $0.15 \mathrm{~m} ; w=0.15 \mathrm{mps} ; \tau_{1}=372.6 \mathrm{~s} ; K_{\mathrm{e}}=0.35 ; K_{\mathrm{y}}=0.36$ during the filtration time, $\tau=$ $3600 \mathrm{~s}$, the dust span decreases from $31 \%$ (for a clean layer) to $17 \%$, which does not correspond to experimental data (see Figure 2, curve 2).

The experimental data on the capture of cement dust better correspond to the empirical dependence obtained by $\mathrm{Yu}$. V. Krasovitsky for the dust of refractory production [23]:

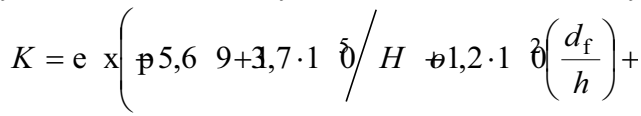

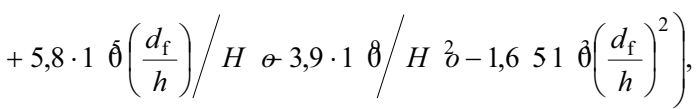

where $H \mathrm{o}=w \tau / d_{f}-$ homochronicity criterion. 2

The formula (13) is obtained for the conditions $10^{5} \leq H \mathrm{o} \leq 3 \cdot 10^{6}, 7.35 \cdot 10^{-3} \leq d_{f} / h \leq 4.3 \cdot 10^{-}$

The time dependence of the cement dust span calculated by formula (13) for the above conditions is shown in Figure 2, curve 3. 
Statistical planning of experimental studies was used to study the patterns of dust capture in cement production by bulk clinker layers. A preliminary search for influencing factors showed that the main factors determining the dust collection processes $d_{g}, w, \delta_{50}, h$, $\mathrm{Z}_{i}$ and $\tau$. Based on these factors, the dominant criteria were identified-Re, Stk, Ho, $d_{f} / h$, $Z_{i} / \rho$.

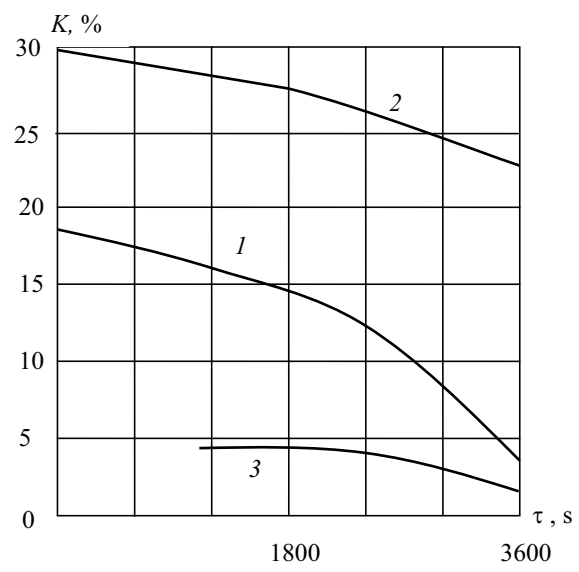

Fig. 2. Dependences of dust span on time, calculated by the formulas: (11) - curve 1; (12) - curve 2; (13) - curve 3

As a result of processing the experimental data set according to a special plan, the dependence of the value of the dust slip through the layer on the main influencing factors was obtained::

$$
K=0,0246\left(\frac{d_{\mathrm{g}}}{d_{\mathrm{g}_{0}}}\right)^{0,245}\left(\frac{w}{w_{0}}\right)^{0,292}\left(\frac{\delta_{50}}{\delta_{0}}\right)^{-0,223}\left(\frac{Z \mathrm{i}}{Z_{\mathrm{b}_{0}}}\right)^{-0,451}\left(\frac{\tau}{\tau_{0}}\right)^{-0,372},
$$

where $d_{\mathrm{g}}=0.01 \mathrm{~m}, w_{0}=0,3 \mathrm{mps} ; \delta_{0}=15^{.10-6} \mathrm{~m} ; \mathrm{Z}_{i 0}=0.01 \mathrm{kgpm}^{3} ; \tau_{0}=1800 \mathrm{~s}$.

Equation (14) is established for the following region of the factor space: $0.005 \leq d_{\mathrm{g}} \leq 0.02$ $\mathrm{m} ; 0.15 \leq w \leq 0.6 \mathrm{mps} ; 7.5 \cdot 10^{-6} \leq \delta_{50} \leq 30 \cdot 10^{-6} \mathrm{~m} ; 0.005 \leq \mathrm{Z}_{i} \leq 0.02 \mathrm{kgpm}^{3} ; 900 \leq \tau \leq 3600 \mathrm{~s}$.

The criterion dependence of the cement dust span, which approximates the results of the experiment, has the form

$$
K=1,43 \cdot 10^{-4} \operatorname{Re}^{0,775} \mathrm{Stk}^{-0,112} H o^{-0,372}\left(\frac{d_{\mathrm{f}}}{h}\right)^{-1,014}\left(\frac{Z_{\mathrm{b}}}{\rho}\right)^{-0,451} .
$$

The influence of the main factors on the amount of dust passage through the bulk clinker filter is shown in Fig.3.

Figure 3 shows that an increase in the average powder size (c) and filtration time (d) independently of other parameters causes an increase in the efficiency of dust cleaning. An increase in the average size of the filter layer filler (a) and the speed of air incoming into the filter (b) reduces the efficiency of dust cleaning. 
$a$

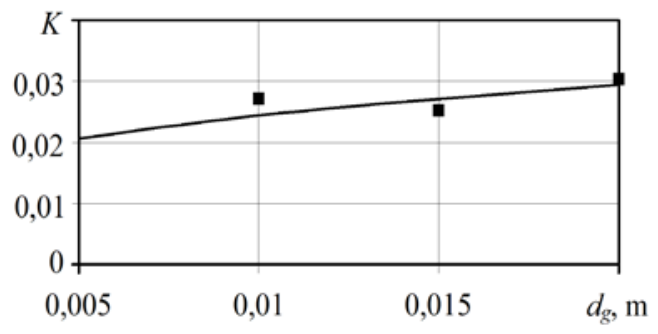

$c$

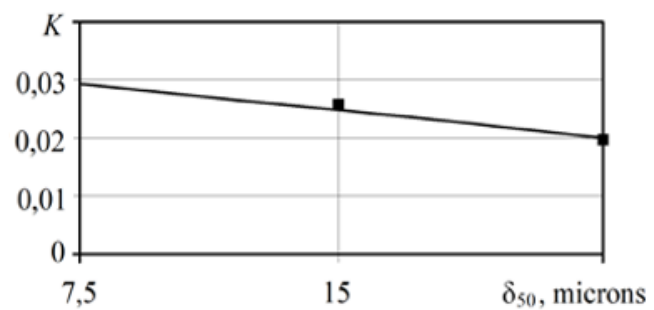

$b$

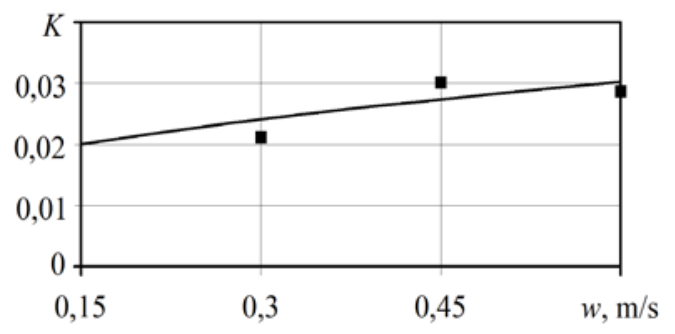

$d$

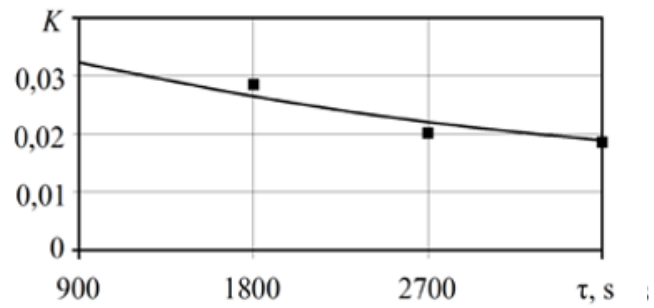

Fig. 3. Dependence of the amount of dust span through the clinker granular layer on the main influencing factors: $a-w=0.3 \mathrm{mps} ; \delta_{50}=15 \mathrm{mkm} ; \tau=1800 \mathrm{~s} ; b-d_{\mathrm{g}}=0,01 \mathrm{~m} ; \delta_{50}=15 \mathrm{mkm} ; \tau=$ $1800 \mathrm{~s} ; c-d_{\mathrm{g}}=0,01 \mathrm{~m} ; w=0,3 \mathrm{mps} ; \tau=1800 \mathrm{~s} ; d-d_{\mathrm{g}}=0,01 \mathrm{~m} ; \delta_{50}=15 \mathrm{mkm} ; w=0,3 \mathrm{mps}:$

- calculation; $\quad$ - experiment

\section{Conclucions}

Based on the experimental data, the criterion dependence of the cement dust slip in the granular filter is obtained. It allows determining the efficiency of the granular filter from its design and technological parameters. It is shown that the obtained expression allows us to more accurately determine the parameters of its operation from the previously developed equations.

\section{References}

1. R. Sharapov, Influence of design and technological parameters on the efficiency of the granular filter, ICMTMTE, (2020).

2. Y. Ovsyannikov, A. Gol'Tsov, A. Seminenko, K. Logachev, V. Uvarov, Reducing the power consumption of ventilation systems through forced recirculation, Refractories and Industrial Ceramics. 57, 5 (2017).

3. Leonard de Clerk, N. Korobova, Russia and the Kyoto Protocol. Metallurgist. 7 (2007).

4. A.Vetoshkin, Theoretical Foundations of Environmental Protection, Mocscow, Higher school, (2008).

5. S. Kabanov, Improving equipment for cleaning dusty gases, Belgorod, BSTU, (2010).

6. M. Aerov, D. Narinsky, Devices with a granular layer. Thermal and hydraulic operation basics. Mocscow, Chemistry, (1979).

7. R. Sharapov, Research of the hydraulic resistance of the granular filter of the sweeping mashnine, E3S Web of Conferences Innovative Technologies in Environmental Science and Education, 135, (2019).

8. Y. Chen, S. Hsiau, C. Hsu, S. Ma, Y. Chang, Influence of operational parameters on 
the performance of gas clean-up technology with a moving granular bed filter Energy, 139, pp. 842-852 (2017).

9. Hot gas filtration - A review Fuel, 104, pp. 83-94. (2013).

10. N. Samokhvalov, Cleaning of industrial emissions from dust by granular filters. Ecology and Industry of Russia № 4. (2009).

11. V. Chumakov, FINGO: effective gas cleaning. Cement and its application.№6. (2006).

12. S. Yankovsky, N. Bulgakova, Means of controlling the dustiness of flows in industrial conditions. Moscow: Tsintikhimneftemash 36 p. (1985).

13. W. Duda, Cement-data-book, Band 1, Internationale Verfahrenstechniken der Zementindustrie, Wiesbaden, Bauverlag, Berlin, p. 617. (1985).

14. P. Elinek, Reconstruction of a clinker cooler dust collection system. Cement and its application. 4 pp. 88-90. (2009).

15. V. Belousov, Theoretical foundations of gas cleaning. M.: Metallurgy 256 p. (1988).

16. P. Raist, Aerosols. Introduction to the theory. M.: Mir, 280 p. (1987).

17. V. Uzhov, Cleaning of industrial gases with electrofilters. M.: Chemistry, $344 \mathrm{p}$. (1967).

18. Yu. Krasovitsky, V. Durov, Dedusting of gases by granular layers. M.: Chemistry 192 p. (1991).

19. R Sharapov, A. Agarkov, Determination of the aerodynamic characteristics of a concentrator with adjustable parameters. MATEC Web of Conferences. $6^{\text {th }}$ International Scientific Conference, (2018).

20. M. Morgulis, M. Mazus, A. Mandrikov, M. Berger, Bag filters. Moscow: Mashinostroenie 256 p. (1977).

21. N. Samokhvalov, E. Skachkov, Method of calculation of bulk granular filters for dust capture. Ecology and Industry of Russia. № 4. pp. 33-35. (2009).

22. N. Samokhvalov, Evaluation of the reliability and durability of granular filters. Construction materials. № 1. C. 28-29. (2007).

23. Yu. Krasovitsky, P. Baltreshas, V. Entin, Dedusting of industrial gases in refractory production. Vilnius: Technika, 364 p. (1996). 